\title{
Madras Motor Neuron Disease: An Interesting Case Report
}

\author{
Naga Srinivas ${ }^{1}$, Sowmini $\mathrm{PR}^{2}$, Kumar Sathish $\mathrm{M}^{3}$, Velayutham Sakthi $\mathrm{S}^{4}$, Jeyaraj Malcolm $\mathrm{K}^{5}$, Saravanan Viveka ${ }^{6}$,
} Mugundhan $\mathrm{K}^{7}$

\begin{abstract}
Madras motor neuron disease (MMND) is a rare childhood/juvenile motor neuron disease reported from various parts of southern India, the first report being from Chennai. This case report is about a young girl who presented with insidious onset, a gradually progressive neurological illness characterized by bilateral sensorineural hearing loss, wasting, and weakness of all four limbs along with bulbar paralysis. On examination, she had involvement of lower cranial nerves, tongue fasciculation, generalized amyotrophy, and bipyramidal features. Electrophysiological studies showed features of chronic denervation. A provisional diagnosis of MMND was made. MMND resembles Brown-Vialetto-Van Laere (BVVL) syndrome and some of the other complex childhood motor neuron disease syndromes, like Boltshauser syndrome, Nathalie syndrome, and Fazio-Londe syndrome. Early diagnosis of BVVL, which is a riboflavin transporter deficiency, is essential, as it is fully responsive to high-dose riboflavin supplementation. In BVVL syndrome, a female predominance has been documented (1:5), while in MMND, an equal distribution is noted. In BVVL, at least half of the reported cases are familial whereas in MMND most of the cases are sporadic. In BVVL, lower motor neuron signs in the limbs are infrequently present and pyramidal signs are rare, whereas in MMND lower and upper motor neuron signs are seen in the majority of the patients. Third, fifth, or sixth cranial nerves are never noted to be affected in MMND, but they may rarely be involved in BVVL. The etiopathophysiology of MMND is still unclear and supportive or symptomatic therapy forms the mainstay of treatment of this orphan disease. Keywords: Madras Motor Neuron Disease, Fasciculation, Sensorineural hearing loss.

Bengal Physician Journal (2021): 10.5005/jp-journals-10070-7050
\end{abstract}

\section{INTRODUCTION}

Madras motor neuron disease (MMND) is a unique childhood motor neuron disease reported first from erstwhile Madras and has a peculiar set of clinical features. Its exact etiopathogenesis still remains elusive, even after 50 years of its recognition. It is classified as an orphan disease due to the small number of cases reported so far. We hope that this case report will throw more light on this rare disease as well as its close differentials.

\section{Case Description}

A 14-year-old thin built girl presented with bilateral sensorineural hearing loss of 9 years duration, thinning involving all four limbs of 2 years duration, and weakness involving both upper limbs as well as both lower limbs of 1-year duration. She had developed difficulty in swallowing food and nasal regurgitation of liquids over the past 6 months. She gave no history of Loss of consciousness (LOC)/seizures/visual disturbances/sensory disturbances. She was born of non-consanguineous parentage and there was no family history of similar illness.

On examination, she was conscious, oriented, and afebrile. Higher mental functions were normal. Examination of cranial nerves revealed bilateral involvement of seventh nerves, eighth nerves, tenth nerves, and twelfth nerves. Bifacial weakness was present. Bilateral profound sensorineural hearing loss was present. Bilateral palatal movements were diminished along with depressed gag reflex. The tongue was atrophied and showed fibrillations (Fig. 1). Generalized wasting was present in all four limbs (Fig. 2). Wasting of all intrinsic muscles in both hands was present (Fig. 3). Pes cavus and hammering of all toes were evident in both feet (Fig. 4). The tone was increased in all four limbs. Distal more than proximal weakness of all four limbs was noted. Bilateral plantar responses were extensor. Other superficial reflexes were normal. Deep tendon reflexes were
${ }^{1-7}$ Department of Neurology, Government Stanley Medical College and Hospital, Chennai, Tamil Nadu, India

Corresponding Author: Mugundhan K, Department of Neurology, Government Stanley Medical College and Hospital, Chennai, Tamil Nadu, India, Phone: +91 9840444280, e-mail: mugundhan69@gmail.com

How to cite this article: Srinivas N, Sowmini PR, Kumar Sathish M, et al. Madras Motor Neuron Disease: An Interesting Case Report. Bengal Physician Journal 2021;8(1):21-23.

Source of support: Nil

Conflict of interest: None

depressed in both upper limbs and exaggerated in both lower limbs. No cerebellar or extrapyramidal features were present.

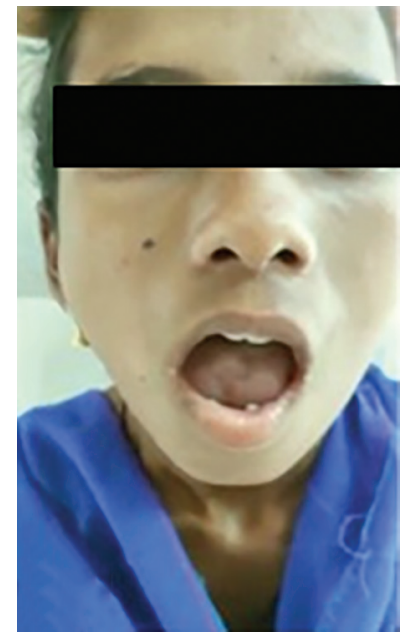

Fig. 1: Tongue atrophy

(c) The Author(s). 2021 Open Access This article is distributed under the terms of the Creative Commons Attribution 4.0 International License (https:// creativecommons.org/licenses/by-nc/4.0/), which permits unrestricted use, distribution, and non-commercial reproduction in any medium, provided you give appropriate credit to the original author(s) and the source, provide a link to the Creative Commons license, and indicate if changes were made. The Creative Commons Public Domain Dedication waiver (http://creativecommons.org/publicdomain/zero/1.0/) applies to the data made available in this article, unless otherwise stated. 


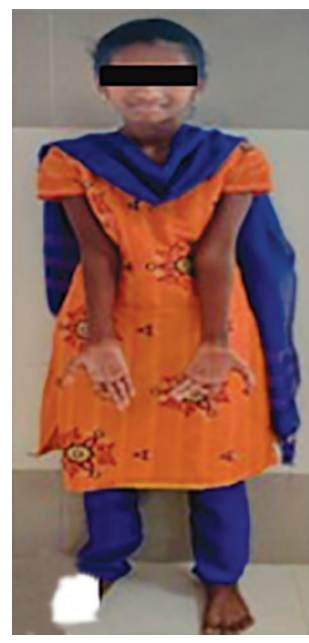

Fig. 2: Generalized wasting was present in all four limbs

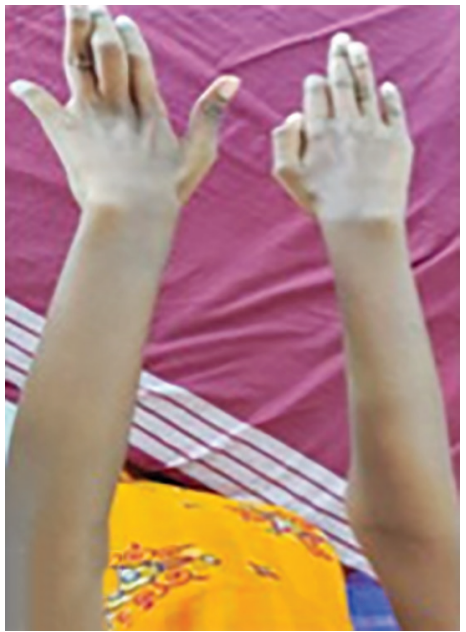

Fig. 3: Wasting of all intrinsic muscles in both hands was present

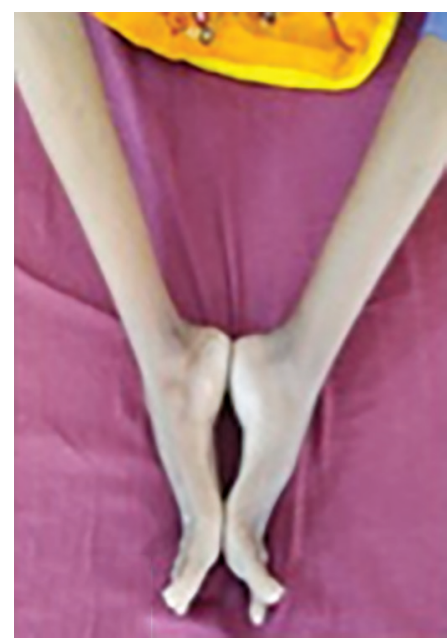

Fig. 4: Pes cavus and hammering of all toes were evident in both feet

Nerve conduction study revealed severe axonal neuropathic changes in all motor nerves, due to gross muscle atrophy with giant $F$ waves. Sensory potentials were normal. Electromyography was done in right deltoid, left abductor pollicis brevis, and right quadriceps muscles that showed no spontaneous activity, high amplitude, long duration, polyphasic motor unit action potentials with reduced recruitment, and incomplete interference, which were suggestive of chronic denervation.

All routine blood investigations were normal. The cardiac evaluation was normal. Ultrasonography of abdomen and pelvis revealed left polycystic ovary. Pure tone audiometry done in both ears showed bilateral profound sensorineural hearing loss. Magnetic resonance imaging brain with the screening of the entire spine was done which was normal.

All the clinical features as well as investigations pointed to a differential diagnosis of MMND versus BVVL syndrome.

\section{Discussion}

MMND was initially described in 1970, by Meenakshisundaram et al. after analyzing the clinical features of 14 patients. This disease entity was described in patients from erstwhile Madras with clinical features of anterior horn cell involvement, facial and bulbar palsy, pyramidal features, and sensorineural hearing impairment. ${ }^{1-3}$

Gourie Devi et al. from NIMHANS, Bangalore presented the clinical findings in 12 cases with juvenile-onset motor neuron disease. The striking clinical and electrophysiological features of these patients were similar to the Madras cases. The male: female ratio was 1:1 in the NIMHANS report while it was 4:1 in the Madras report. One-third of Bangalore patients had polyminimyoclonus in fingers. ${ }^{4,5}$

Reduced citrate and high pyruvate values have been reported in patients with MMND. ${ }^{6}$

Electrocochleography and brain auditory evoked potential done on these cases suggest loss of auditory nerve fibers and/or sensory cells in the spiral ganglion as the probable cause for the sensorineural deafness. ${ }^{7}$

MMND is a very rare entity with only 150 reported cases all over the world, most of whom are from South India. Madras motor disease was described initially as a sporadic form of benign childhood motor neuron disease. Several reports of familial MMND were described a few years later with an autosomal recessive form of inheritance. The patients who had optic atrophy as an additional feature were classified to have MMND variant, which constitutes more than a quarter of the total reported cases of MMND.

BVVL syndrome, Boltshauser syndrome, Nathalie syndrome, and Fazio-Londe syndrome are the common anterior horn cell diseases mimicking MMND.

BVVL syndrome has similar clinical features as that of MMND, but is caused due to mutations in SLC52A2 (encoding RFVT2) or SLC52A3 (encoding RFVT3) which produces riboflavin transporter defect that improves with riboflavin therapy thereby preventing the progression of this rare neurodegenerative condition..$^{8,9}$ In MMND, a combination of lower and upper motor neuron signs are seen and third or sixth cranial nerves are never affected differentiating it from BVVL. ${ }^{10}$

In BVVL syndrome, females are commonly affected (1:5) and most cases are familial whereas in MMND most of the cases are sporadic. Some case reports hypothesize the inflammatory pathogenesis in MMND. An unknown expanded repeat of C9ORF72 may possibly play a role. However, a combination of genetic and environmental factors is thought to play causative roles. The familial cases of MMND may probably share a common biological pathway with BVVL ${ }^{11}$ (Flowchart 1). 
Flowchart 1: Approach to motor neuron disease with sensorineural deafness

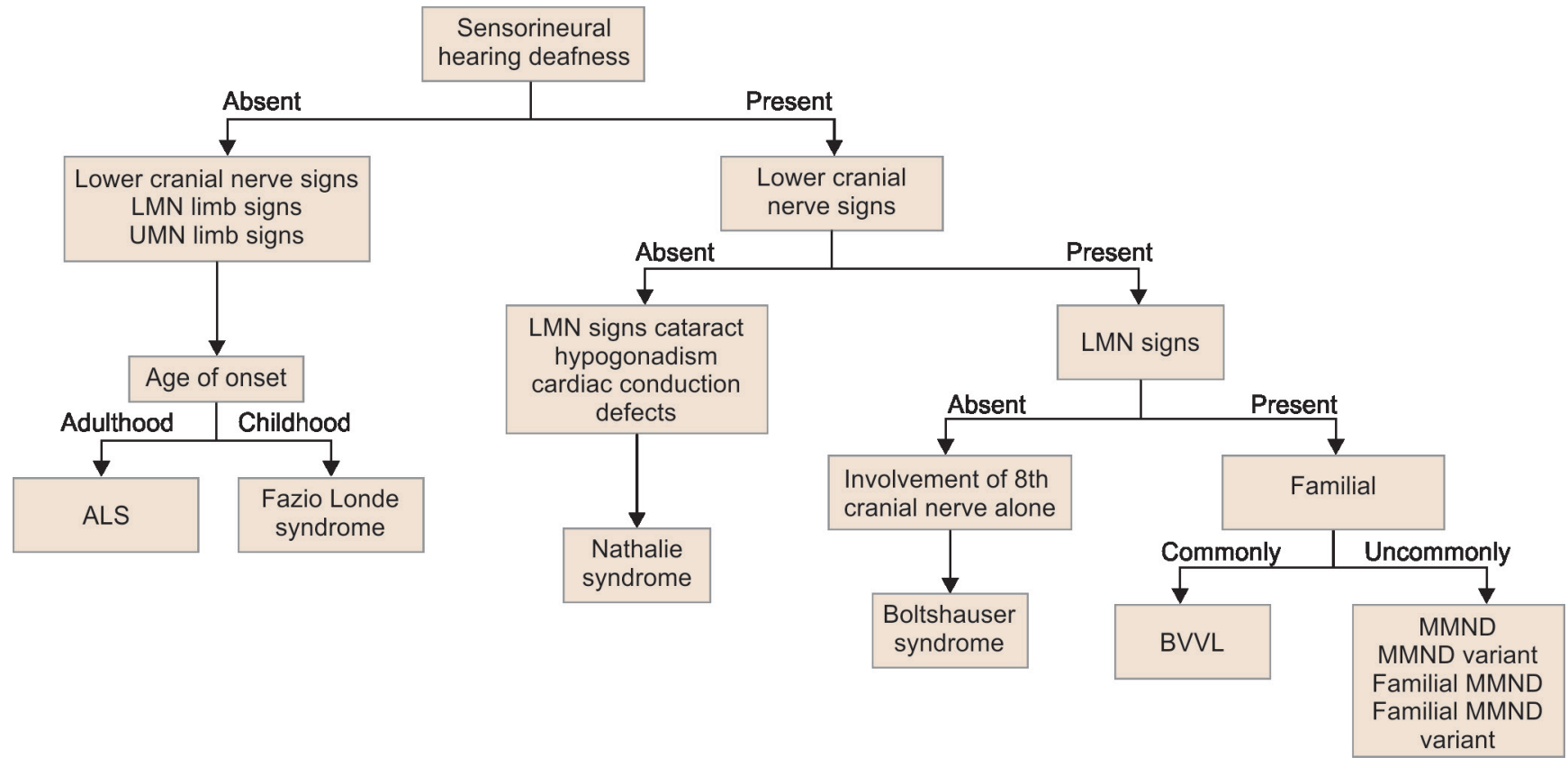

There is no specific treatment for MMND. However, supportive and symptomatic therapy like hearing aids may help these patients. The patients with MMND usually have a normal life span.

Our patient was advised genetic screening for SLC52A2 and SLC52A3 genes to rule out BVVL, but she was not willing to do the tests. However, we tried high-dose riboflavin supplementation for 3 months as a therapeutic trial, but no improvement was noted. This young girl with MMND is on regular follow-up.

\section{References}

1. Meenakshisundaram E, Jagannathan K, Ramamurthi B. Clinical pattern of motor neuron disease seen in younger age groups in Madras. Neurol India 1970;18(Suppl. 1):109. Available at: https:// pubmed.ncbi.nlm.nih.gov/5508105/

2. Jagannathan K. Juvenile motor neuron disease. In: Spillane JD, editor. Tropical neurology. London: Oxford Univ Press; 1973, pp. 127-130.

3. Jagannathan K, Kumaresan G. Madras pattern of motor neuron disease. In: Gourie-Devi M, editor. Motor neuron disease. New Delhi: Oxford and IBH; 1987, pp. 191-193.

4. Gourie-Devi M, Suresh TG. Madras pattern of motor neuron disease in South India. J Neurol Neurosurg Psychiatry 1988;51(6):773-777. DOI: 10.1136/jnnp.51.6.773.
5. Gourie-Devi M, Suresh T, Shankar S. Pattern of motor neuron disease in South India and Monomelic amyotrophy (a benign atypical form) In: Gourie-Devi M, editor. Motor neuron disease. New Delhi: Oxford and IBH; 1987, pp. 171-190.

6. Valmikinathan K, Mascreen M, Meenakshisundaram E, et al. Biochemical aspects of motor neurone disease-Madras pattern. J Neurol Neurosurg Psychiatry 1973;36(5):753-756. DOI: 10.1136/ jnnp.36.5.753.

7. Wadia PN, BhattMH, Misra VP. Clinical neurophysiological examination of deafness associated with juvenile motor neurone disease. J Neurol Sci 1987;78:29-33. DOI: 10.1016/0022-510x(87)90075-x.

8. Sathasivam S. Brown-Vialetto-Van Laere syndrome review. Orphanet J Rare Dis 2008;3:9. DOI: 10.1186/1750-1172-3-9.

9. Kranthi P, Garuda BR, Gopi S, et al. Brown-Vialetto-Van Laere syndrome: a rare case report of MND mimic. Neurology 2020;68(5):1217-1219. DOI: 10.4103/0028-3886.299175.

10. Nalini A, Thennarasu K, Yamini BK, et al. Madras motor neuron disease (MMND): clinical description and survival pattern of 116 patients from Southern India seen over 36 years (1971-2007). J Neurol Sci 2008;269(1-2):65-73. DOI: 10.1016/j.jns.2007.12.026.

11. Nalini A, Pandraud A, Mok K, et al. Madras motor neuron disease (MMND) is distinct from the riboflavin transporter genetic defects that cause Brown-Vialetto-Van Laere syndrome. J Neurol Sci 2013;334:119-122. DOI: 10.1016/j.jns.2013.08.003. 